\title{
A Novel Case of Orofacial Pain Treated by Dry Needling Technique - A Case Report
}

\author{
Asha V, Raj Kannan and Thanuja Raju Jacob*
}

Department of Oral Medicine and Radiology, The Oxford Dental College and Hospital, Bommenhalli, Bangalore, Karnataka, India

\begin{abstract}
Temporomandibular disorders (TMDs) are a subgroup of orofacial pain which cause a significant public health problem affecting approximately $5-12 \%$ of the population. TMD is manifested by pain, joint sounds, and limitation in jaw movement, muscle tenderness and joint tenderness. Myogenous TMDs are characterized by pain and dysfunction that arise from pathologic and functional process in the masticatory muscles. Myofascial trigger points are one of the diagnostic criteria for the most common pain related TMDs. The current case report highlights the importance of considering the posture, locating the myofascial trigger point within the muscles and the effectiveness of Dry Needling Technique in resolving non-odontogenic causes of orofacial pain.
\end{abstract}

Keywords: Myofascial pain; Trigger points; Dry needling; Orofacial pain

\section{Introduction}

Orofacial pain refers to a large group of disorders, including temporomandibular disorders (TMDs), headaches, neuralgia, pain arising from dental or mucosal origin and idiopathic pain $[1,2]$. Due to the diverse nature of the causes of orofacial pain, it often poses challenge to the health professionals to diagnose and treat this disorder. TMDs are a subgroup of orofacial pain which can cause a significant public health problem affecting approximately $5-12 \%$ of the population [3]. TMD is manifested by pain, joint sounds, and limitation in jaw movement, muscle tenderness and joint tenderness. It is also associated with other symptoms affecting the head and neck region such as headache, earrelated symptoms and cervical spine disorders [4].

Myogenous TMDs are characterized by pain and dysfunction that arise from pathologic and functional process in the masticatory muscles. Masticatory myofascial pain is also included in the taxonomic classification in recent diagnostic criteria of TMD validation project [5]. Myofascial trigger points are one of the diagnostic criteria for the most common pain related TMDs. A Myofascial trigger point (MTrP) is defined as a hyperirritable spot in skeletal muscle that is associated with a hypersensitive palpable nodule in a taut band. The spot is painful on compression and can give rise to characteristic referred pain, referred tenderness, motor dysfunction, and autonomic phenomena [6]. Myofascial pain must be suspected in patients with pain in the masticatory muscles located in the preauricular or mandibular area, frontal or temporal regions, or in the ear, along with the existence of painful myofascial trigger points on palpation [7]. The causes of these MTrPs are attributed to repetitive stress to the masticatory muscles like in parafunctional habits, secondary to malocclusion, postural problems involving the cervical spine and temporomandibular joint. Sometimes MTrPs could result from mechanical stress like sudden abrupt movements, motor vehicle accidents, falls, fractures, joint sprains or dislocations, a direct blow to a muscle, joint, or mandible, excessive exercise or activity or performing new or unusual activities [8]. There are studies done on involvement of Masticatory muscles MTrPs in Orofacial pain and its clinical presentations are quite varied in nature and hence the treatment options.

Treatments of MTrPs are aimed at deactivating it and thereby restoring the functional goals of the patients. This can be done by trigger point release, spray and stretch, post isometric relaxation, dry needling or sometimes combination of two or three methods. Dry needling uses a thin filiform needle to penetrate the skin and stimulate underlying myofascial trigger points, muscular, and connective tissues for the management of neuro-musculoskeletal pain and impairment in movement [9]. The advantages of dry needling are increasingly documented and include an immediate reduction in local, referred, and widespread pain, restoration of range of motion and muscle activation patterns, and a normalization of the immediate chemical environment of active myofascial trigger points [10]. The purpose of this case report is to imply the importance of considering myofascial trigger points in orofacial pain including ear pain and to support the efficacy of dry needling technique in the management of such pain.

\section{Case Description}

A 29 year old female patient came to the department of Oral Medicine \& Radiology, with a chief complaint of pain over left preauricular and left ear for the past 3 months. She had no other relevant history of trauma or infection. The pain was dull, diffuse and continuous in nature and it also referred to the left temporal region. She was also experiencing headache from the same duration. The pain was aggravating on chewing food and also disturbing her sleep. She was a tailor by profession for the past ten years. She first consulted an ENT specialist who cleared her of any ear dysfunctions however she was put on muscle relaxants for seven days. The pain subsided on taking the medication but aggravated soon after the course was over. She was referred to a neurologist and was advised for a CT scan of the head which revealed no abnormalities.

The patient was admitted into the Department of Oral Medicine and Radiology for further evaluation and management.

On pain assessment, the pain was nine in numeric pain rating scale. The patient was primarily concerned about her left ear pain. On extra oral examination, there was no swelling over the temporomandibular

*Corresponding author: Thanuja Raju Jacob, MDS, The Oxford Dental College and Hospital, Bommenhalli, Bangalore, Karnataka, India-560068; Tel: +91 9916872907; E-mail: r.tanu.315@gmail.com

Received June 08, 2015; Accepted July 08, 2015; Published July 15, 2015

Citation: Asha V, Kannan R, Jacob TR (2015) A Novel Case of Orofacial Pain Treated by Dry Needling Technique - A Case Report. Dentistry 5: 319. doi:10.4172/2161-1122.1000319

Copyright: ( 2015 Asha V, et al. This is an open-access article distributed under the terms of the Creative Commons Attribution License, which permits unrestricted use, distribution, and reproduction in any medium, provided the original author and source are credited. 
region. The maximum mouth opening was limited to $21 \mathrm{~mm}$, above which she could not open her mouth due to pain (Figure 1).

There was no mandibular deviation, clicking sounds or any other symptoms related to disc dysfunction. Facial symmetry was normal. No para functional habits were observed or reported. Clench test and provocation test were negative for TMJ problem [5].

On palpation there was a marked tenderness over the left masseter, lateral pterygoid, medial pterygoid, temporalis, sternocleidomastoid, digastric and suboccipital muscles. The tenderness was also observed in the opposite side but with less intensity. The palpation revealed the presence of myofascial trigger points over her masticatory and suboccipital muscles. Palpation of masseter referred pain to the left ear. The taut band, exquisite tenderness and referral pain were all quite indicative of myofascial pain [6] (Figure 2).

On further investigations the OPG and $\mathrm{T}$ scan revealed no abnormalities. Based on the history and examination, the patient was diagnosed as myofascial pain syndrome in the orofacial region.

The patient was treated with dry needling technique by an experienced physiotherapist with specialized training in dry needling technique. Dry needling is a new form of treatment for myofascial pain syndrome. The aim of the treatment was to deactivate the myofascial trigger points in the orofacial muscles. Before the session appropriate precautions were taken and contraindications were checked. An informed consent form was signed by the patient and she was explained about the procedure and its expected outcomes.

In the first session, she was needled for sternocleidomastoid and masseter using a $25 \mathrm{~mm}$ stainless steel needle. The muscles were palpated to identify the exact location of the MTrPs and then needle was angled perpendicular to the point. Lift and thrust method was used with the aim of eliciting the Local Twitch Response (LTR). LTR is a brisk contraction of a group of muscle fibers in a taut band, in response to a rapid, brief mechanical stimulation at the active MTrP site [6]. The session lasted for ten minutes followed by stretching of those needled muscles. TMD index questionnaire [11] was asked and the patient reported $65 \%$ disability. The patient was asked to self-release the needled muscles as well as digastric and suboccipital muscles, twice a day followed by stretch. She was asked to do the same on muscles of both sides of the face.

On the second visit, the patient came with $50 \%$ reduction in pain and her mouth opening increased to $30 \mathrm{~mm}$. Her headache was also considerably reduced in intensity and frequency. Dry needling for lateral pterygoid, temporalis and deep masseter muscles was done. Needles of the sizes $40 \mathrm{~mm}$ and $13 \mathrm{~mm}$ were used for lateral pterygoid and temporalis respectively. LTR was elicited for the masseter. Total duration of needling was fifteen minutes. After needling, stretching was done and the patient was asked to continue the home exercises.

On the third visit on the seventh day, the patient came with complete resolution of the pain. Her mouth opening increased. Headache was not felt any longer after the second session. Tenderness was absent and she felt no pain upon palpating the sternocleidomastoid, masseter, digastric and suboccipital muscles. TMD index questionnaire reported no disability (Table 1).

The patient was asked to do regular postural correction exercises to the neck and do self-release and stretches at home. She was followed after three months and she remained completely painless.

\section{Discussion}

The present case report reveals the importance of considering myofascial pain while treating patients with orofacial pain. Dry needling technique could be a viable option to resolve this pain. Myofascial pain is one of the major causes of non-dental pain in orofacial region $[12,13]$. In this current case report, the patient was diagnosed with myofascial pain syndrome of the orofacial region. Trigger points were identified bilaterally over masseter, temporalis, lateral pterygoid and medial pterygoid, suboccipital muscles and sternocleidomastoid muscles. These muscles were more painful in the left side compare to the right side of the face.

The cause of the pain is varied and the etiology could be her altered posture due to her job as a tailor where she used to keep her head flexed. Forward head posture (FHP) is associated with TMDs $[14,15]$ and many authors have suggested that postural problems may lead to pain and dysfunction, while others postulated that FHP is associated with TMDs. In FHP, the head moves front leading to upper cervical extension and lower cervical flexion. This forward movement reciprocally stretches the suprahyoid muscles than tends to pull the

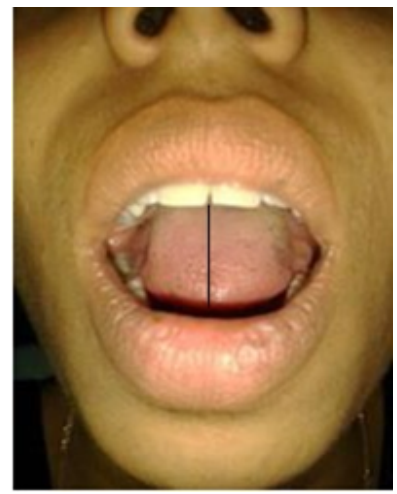

Figure 1: The maximum mouth opening was limited to $21 \mathrm{~mm}$.

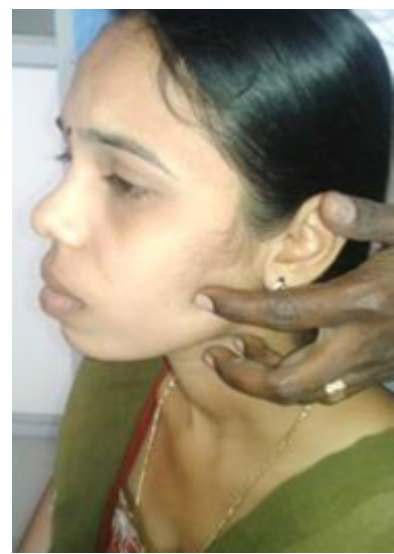

Figure 2: Palpation of masseter referred pain to the left ear.

\begin{tabular}{|c|c|c|c|}
\hline & First visit & Second visit & Final visit \\
\hline Numeric Pain Rating scale & 9 & 4 & 0 \\
\hline Maximal Mouth Opening & 21 & 30 & 38 \\
\hline TMD Index Questionnaire & $65 \%$ disability & $20 \%$ disability & $0 \%$ disability \\
\hline
\end{tabular}

Table 1: Results of patient's response in three sessions of dry needling technique. 
mandible inferiorly. This movement must be countered by mandibular elevators like temporalis and masseter. Thus those muscles harbor MTrPs. Apart from masticatory muscles; even the cervical muscles like sternocleidomastoid and suboccipital were also involved. These two are the muscles that work constantly to maintain the forward head posture.

Though these muscles were painful on palpation, the patient's major complaint was pain in the left ear. This is understandable as the Masseter and lateral pteregoid pain referral zone is over the ear. Salvetti, et al. reported that the prevalence of ear symptoms in the general population is varied from $10 \%-31 \%$, and increases up to $85 \%$ in TMD patients [16]. This could be due to the common innervation to both these regions by trigeminal nerve. Tensor tympani, a muscle in the middle ear, can become sensitized with repetitive pain stimulation due to masticatory myofascial pain. This can be a source of ear pain, ear fullness or even temporary hearing loss [17]. This strongly suggests that the patient's ear pain could be due to a myofascial source and in this case we treated the myofascial trigger points. This was confirmed as the patient's ear pain reduced when we treated those muscles.

For the successful management of MTrP pain, it is essential to first identify all and deactivate them [18]. This can be done by various noninvasive methods like manual trigger point compression, spray and stretch, strain/counter strain, ultrasound and various forms of muscle stretching [19]. Dry needling is a new form of treatment, where a thin solid filament needle is inserted into the trigger point to deactivate it.

A recent systematic review showed that there is a strong evidence for dry needling to have a positive effect on pain intensity [20]. Previously studies were done on masseter and lateral pterygoid dry needling and the results were very encouraging [21,22]. In our patient, we have given two sessions of dry needling treatment over masseter, lateral pterygoid, temporalis and sternocleidomastoid. We used deep dry needling with lift and thrust maneuver over the taut band of the muscles. This is the common method employed to deactivate the trigger points.

This case report shows the effect of dry needling technique in orofacial pain. Dry needling is cost effective, easy to learn with appropriate training, carrying lower risk and minimally invasive treatment modality [23]. So many theories explain the pain reduction following dry needling [24-31]. The most common theory as postulated by Simons and Travell was the disruption of the MTrP contraction knots [6]. It even destroys the motor end plates and causes distal axon denervation when the needle hit an MTrP. The literature claims that it changes the end plate cholinesterase and Ach receptors as part of the normal muscle regeneration process [32]. Needling destroys the existing MTrPs and allows the loci to regenerate in a normal manner which in one way relieves the pain. Other theories state that dry needling having the segmental anti-nociceptive effects [33]. Trigeminal nerve is the common neural pathway for masticatory muscles and ear. Hence needle stimulation at masticatory region can have a positive impact on the ear symptoms, due to segmental anti-nociception. One more proposed mechanism is the connection the lateral pterygoid has to the malleus in the middle ear through the superior retrodiscal lamina. So dry needling directed to that muscle could have resolved the ear symptoms. Other effects of dry needling include immediate reduction in pain due to descending inhibitory system [34]. This is achieved by rapidly moving the needle within the MTrPs which result in hyper stimulation analgesia for general pain control. Our patient's improvement in ear pain and headache could be due to any or all of the above mentioned factors.

The current case report highlights the importance of considering the posture, locating the myofascial trigger point within the muscles and the effectiveness of dry needling technique in resolving nonodontogenic causes of orofacial pain. However the contraindications include extreme fear of needles, presence of local or systemic infections and acute muscle trauma [35]. By the dry needling technique, home exercise regimen and postural correction the patient recovered completely. Dry needling is a great technique to try out if the patients have identifiable MTrPs which could be clinically correlated with the patient's current symptoms.

\section{References}

1. Agostoni E, Frigerio $R$, Santoro $P$ (2005) Atypical facial pain: clinical considerations and differential diagnosis. Neurol Sci 26: s71-s74.

2. Madland G, Newton-John T, Feinmann C (2001) Chronic idiopathic orofacial pain: I: What is the evidence base? Br Dent J 191: 22-24

3. http://www.nidcr.nih.gov/DataStatistics/FindDataByTopic/FacialPain/

4. de Wijer A, de Leeuw JR, Steenks MH, Bosman F (1996) Temporomandibular and cervical spine disorders: self-reported signs and symptoms. Spine 21 : 1638-1646.

5. Schiffman E, Ohrbach R, Truelove E, Look J, Anderson G, et al. (2014) Diagnostic Criteria for Temporomandibular Disorders (DC/TMD) for Clinical and Research Applications: recommendations of the International RDC/TMD Consortium Network and Orofacial Pain Special Interest Group. J Oral Facial Pain Headache 28: 6-27.

6. Travell JG, Simons DG (1999) Travell and Simons' myofascial pain and dysfunction; the trigger point manual. ( $2^{\text {nd }}$ edn), Lippincott Williams \& Wilkins, Baltimore.

7. Gonzalez-Perez LM, Infante-Cossio P, Granados-Nuñez M, Urresti-Lopez FZ (2012) Treatment of temporomandibular myofascial pain with deep dry needling. Med Oral Patol Oral Cir Bucal 17: e781-e785.

8. Dommerholt J (2006) Myofascial pain syndrome in craniomandibular region In: Padrós Serrat E. (ed) Bases diagnosticas, terapeuticas y posturales de functionalismo craniofacial. Madrid, Ripano

9. APTA (2013) Description of dry needling in clinical practice: an educational resource paper. APTA Public Policy, Practice, and Professional Affairs Unit.

10. Dommerholt $J$ (2011) Dry needling - peripheral and central considerations. $J$ Man Manip Ther 19: 223-227.

11. http://www.mcphysicaltherapy.com/pdf/TMJ.pdf

12. McNeil C (1993) Temporomandibular disorders: guidelines for classification, assessment and management. ( $2^{\text {nd }}$ edn), Quintessence Publishing Company, Chicago.

13. Moss RA, Ruff MH, Sturgis ET (1984) Oral behavioral patterns in facial pain headache and non headache populations. Behav Res Ther 22: 683-697.

14. Lee WY, Okeson JP, Lindroth J (1995) The relationship between forward head posture and temporomandibular disorders. J Orofac Pain 9: 161-167.

15. Ciancaglini R, Testa M, Radaelli G (1999) Association of neck pain with symptoms of temporomandibular dysfunction in the general adult population. Scand J Rehabil Med 31: 17-22.

16. Salvetti G, Manfredini D, Barsotti S, Bosco M (2006) Otologic symptoms in temporomandibular disorders patients: is there evidence of an associationrelationship? Minerva Stomatol 55: 627-637.

17. Dalla-Bona D, Shackleton T, Clark G, Ram S (2015) Unilateral ear fullness and temporary hearing loss diagnosed and successfully managed as temporomandibular disorder: A case report. J Am Dent Assoc 146: 192-194.

18. Baldry $P$ (2002) Management of myofascial trigger point pain. Acupunct Med 20: $2-10$.

19. Mense S, Gerwin RD (2010) Muscle pain: diagnosis and treatment. SpringerVerlag Berlin Heidelberg.

20. Cagnie B, Castelein B, Pollie F, Steelant L, Verhoeyen H, et al. (2015) Evidence for the use of ischemic compression and dry needling in the management of trigger points of the upper trapezius in patients with neck pain: a systematic review. Am J Phys Med Rehabil 94: 573-583. 
Citation: Asha V, Kannan R, Jacob TR (2015) A Novel Case of Orofacial Pain Treated by Dry Needling Technique - A Case Report. Dentistry 5: 319. doi:10.4172/2161-1122.1000319

21. Gonzalez-Perez LM, Infante-Cossio P, Granados-Nunez M, Urresti-Lopez FJ, Lopez- Martos R, et al. (2015) Deep dry needling of trigger points located in the lateral pteregoid muscle: Efficacy and safety of treatment for management of myofascial pain and temporomandibular dysfunction. Med Oral Patol Oral Cir Bucal 20: e326-e333.

22. Fernandez-Carnero J, La Touche R, Ortega-Santiago R, Galan-del-Rio F, Pesquera J, et al. (2010) Short -term effects of dry needling of active myofascial trigger points in the masseter muscle in patients with temporomandibular disorders. J Orofac Pain 24: 106-112.

23. Kalichman L, Vulfsons S (2010) Dry needling in the management of musculoskeletal pain. J Am Board Fam Med 23: 640-646.

24. Fricton $J$ (2007) Myogenous temporomandibular disorders: diagnostic and management considerations. Dent Clin North Am 51: 61-83.

25. http://www.practicalpainmanagement.com/pain/maxillofacial/tmj/tmd-facialpain-forward-head-posture

26. Sola AE, Bonica JJ (1990) Myofascial pain syndromes. In: Bonica JJ, Loeser JD, Chapman CR, Fordyce WE. (eds.) The management of pain. (2nd edn), Lea \& Febiger, Philadelphia.

27. Rachlin ES (1994) Myofascial pain and fibromyalgia: trigger point management. Mosby, St. Louis.
28. Hong CZ (1994) Lidocaine injection versus dry needling to myofascial trigge point. The importance of the local twitch response. Am J Phys Med Rehabil 73: 256-263.

29. Ruoff GE (1995) Technique of trigger point injection. In: Pfenninger JL, Fowler GC. (eds.) Procedures for primary care physicians. Mosby, St. Louis.

30. Fischer AA (1997) New approaches in treatment of myofascial pain. Phys Med Rehabil Clin North Am 8: 153-169.

31. Zohn DA, Mennell JM (1976) Clinical examination. In: Musculoskeletal pain: diagnosis and physical treatment. Little, Brown, Boston.

32. Sadeh M, Stern LZ, Czyzewski K (1985) Changes in end-plate cholinesterase and axons during muscle degeneration and regeneration. J Anat 140: 165-176.

33. Srbely JZ, Dickey JP, Lee D, Lowerison M (2010) Dry needle stimulation of myofascial trigger points evokes segmental anti-nociceptive effects. J Rehabil Med 42: 463-468.

34. Melzack R (1981) Myofascial trigger points: relation to acupuncture and mechanisms of pain. Arch Phys Med Rehabil 62: 114-117.

35. Alvarez DJ, Rockwell PG (2002) Trigger points: diagnosis and management. Am Fam Physician 65: 653-660. 